\title{
Immersive Shared Experiences
}

\author{
Pablo Cesar \\ CWI: Centrum Wiskunde \& Informatica \\ Science Park 123, 1098 XG Amsterdam, Netherlands \\ p.s.cesar@cwi.nl
}

\begin{abstract}
Video communication is filtering into everyday use. Television is becoming more social, videoconferencing happens in the living room and beyond, and games are played with others remotely. The confluence of social networking, multimedia, and computermediated interaction is radically reshaping social communication, bringing new challenges and opportunities. One key challenge ahead is to move from current static solutions (e.g., "talking heads") to truly natural and immersive experiences. In this talk we provide an overview of the present state of the art through a few examples and discuss future possibilities and challenges. In particular, we will concentrate on two representative future application areas: performing arts and personal communication. In the former, we will explore how distributed stages can be linked, so artists at different locations can perform together at the same time the same song, act or play. In the latter, we will discuss our continuous efforts on the provision of 3D tele-immersion environments that offer a common virtual room for friends or family members to hangout. As we will show in this talk, current challenges associated to immersive media experience require multi-disciplinary solutions.
\end{abstract}

\section{Categories and Subject Descriptors}

H.4 [Information Systems Applications]: Communications Applications; H.5 [Information Interfaces and Presentation (e.g. $\mathrm{HCI}$ ]: multimedia information systems, user interfaces, hypertext, hypermedia; I.3 [Computer Graphics]: ThreeDimensional Graphics and Realism

\section{General Terms}

Measurement, Design, Experimentation, Human Factors.

\section{Keywords}

Immersive Media; Socially-Aware Multimedia; Media Synchronization; Shared Experiences.

Permission to make digital or hard copies of part or all of this work for personal or classroom use is granted without fee provided that copies are not made or distributed for profit or commercial advantage and that copies bear this notice and the full citation on the first page. Copyrights for third-party components of this work must be honored. For all other uses, contact the Owner/Author.

Copyright is held by the owner/author(s).

ImmersiveME'15, October 30-30 2015, Brisbane, Australia

ACM 978-1-4503-3745-8/15/10.

http://dx.doi.org/10.1145/2814347.2814355

\section{BIO}

Dr. Pablo Cesar leads Distributed and Interactive Systems group (http://www.dis.cwi.nl) at CWI. Pablo's research focuses on modeling and controlling complex collections of media objects (including real-time media and sensor data) that are distributed in time and space. His fundamental interest is in understanding how different customizations of such collections affect the user experience. In particular, he is interested about the following research questions:

- Dynamic media creation and production: facilitating temporal compilations and aggregations based on the dynamic constraints (preferences, context);

- User response and attention: development of mechanisms for monitoring and modeling user affinity and engagement to events and media content; and

- Social and immersive consumption of media: policies and interfaces for content adaptation.

Pablo is the PI of a Public Private Partnership project with Xinhuanet and of the very successful EU-funded projects REVERIE and Vconect. He has (co)-authored over 100 articles. $\mathrm{He}$ is member of the editorial board of, among others, ACM Transactions on Multimedia (TOMM). Pablo has given tutorials about multimedia systems in prestigious conferences such as ACM Multimedia, CHI, and the WWW conference. He acted as an invited expert at the European Commission's Future Media Internet Architecture Think Tank and participates in standardisation activities at MPEG (point-cloud compression) and ITU (QoE for multi-party tele-meetings).

More information: http://homepages.cwi.nl/ garcia/

\section{REFERENCES}

[1] P. Cesar, R. Kaiser, and M.F. Ursu. 2014. Toward Connected Shared Experiences. IEEE Computer, 47(7): 86-89.

[2] R. Mekuria, M. Sanna, E. Izquierdo, D.C.A. Bulterman, and P. Cesar. 2014. Enabling 3D Tele-Immersion with Live Reconstructed Mesh Geometry with Fast Mesh Compression and Linear Rateless Coding. IEEE Transactions on Multimedia (TMM), 16(7): 1809-1820.

[3] M. Obrist, P. Cesar, D. Geerts, T. Bartindale, and E.F. Churchill. 2015. Online Video and Interactive TV Experiences - A Growing Research Field. ACM Interactions.

[4] D. Williams, P. Cesar, M.F. Ursu, I. Kegel, J. Jansen, and E. Geelhoed. 2015. Experiments with Distributed Theatre. IEEE MultiMedia (IEEE MM), 22(3): 4-9. 